\title{
DETECTION AND MAPPING OF DECOUPLED STELLAR AND IONIZED GAS STRUCTURES IN THE ULTRALUMINOUS INFRARED GALAXY IRAS $12112+0305^{1}$
}

\author{
Luis Colina, ${ }^{2}$ Santiago Arribas, ${ }^{3,4}$ Kirk D. Borne, ${ }^{5}$ and Ana Monreal ${ }^{3}$ \\ Received 2000 January 31; accepted 2000 February 28; published 2000 March 16
}

\begin{abstract}
Integral field optical spectroscopy with the INTEGRAL fiber-fed system and Hubble Space Telescope optical imaging are used to map the complex stellar and warm ionized gas structure in the ultraluminous infrared galaxy IRAS 12112+0305. Images reconstructed from wavelength-delimited extractions of the integral field spectra reveal that the observed ionized gas distribution is decoupled from the stellar main body of the galaxy, with the dominant continuum and emission-line regions separated by projected distances of up to $7.5 \mathrm{kpc}$. The two optical nuclei are detected as apparently faint emission-line regions, and their optical properties are consistent with being dust-enshrouded weak [O I] LINERs. The brightest emission-line region is associated with a faint $\left(m_{I}=20.4\right)$, giant $\mathrm{H}$ II region of $600 \mathrm{pc}$ diameter, in which a young ( $\sim \mathrm{Myr})$ massive cluster of about $2 \times 10^{7} M_{\odot}$ dominates the ionization. Internal reddening toward the line-emitting regions and the optical nuclei ranges from 1 to 8 mag in the visual. Taking the reddening into account, the overall star formation in IRAS $12112+0305$ is dominated by starbursts associated with the two nuclei and corresponds to a star formation rate of $80 M_{\odot} \mathrm{yr}^{-1}$.
\end{abstract}

Subject headings: galaxies: active — galaxies: individual (IRAS 12112+0305) — galaxies: interactions — galaxies: nuclei - galaxies: starburst

\section{INTRODUCTION}

Ultraluminous infrared galaxies (ULIRGs), with bolometric luminosities $L_{\mathrm{bol}} \approx L_{\mathrm{IR}} \geq 10^{12} L_{\odot}$, are the most luminous galaxies in the local universe. ULIRGs show signs of strong interactions and mergers (Melnick \& Mirabel 1990; Clements et al. 1996; K. D. Borne, H. Bushouse, L. Colina, \& R. A. Lucas 2000 , in preparation) or even multiple collisions (Borne et al. 2000), and they have large amounts of gas and dust that significantly obscure the nuclear ionizing sources (Sanders \& Mirabel 1996). ULIRGs appear to be the low-redshift analogs of the high-redshift submillimeter galaxies (SCUBA sources), which are responsible for the bulk of the far-infrared background radiation (see Sanders 2000 for a review).

Mid-infrared spectroscopy has shown that ULIRGs with optical H II- and LINER-like spectra are dominated by starbursts (Lutz et al. 1998; Genzel et al. 1998; Lutz, Veilleux, \& Genzel 1999). However, the increased fraction of optically classified Seyfert galaxies among the more luminous ULIRGs $\left(\log L_{\text {IR }} \geq 12.3 L_{\odot}\right)$ is taken as evidence for the presence of dust-enshrouded quasars powering these galaxies (Veilleux, Kim, \& Sanders 1999a).

The ionization structure of ULIRGs is extended and rather complex, and therefore narrow-slit $\left(\leq 2^{\prime \prime}\right)$ optical spectroscopy along a given position angle cannot adequately indicate the location, nature, and interplay of the different ionization mechanisms present in these galaxies. The spectral classification of

\footnotetext{
${ }^{1}$ Based on observations with the William Herschel Telescope operated on the island of La Palma by the ING in the Spanish Observatorio del Roque de los Muchachos of the Instituto de Astrofísica de Canarias. Based also on observations with the NASA/ESA Hubble Space Telescope, obtained at the Space Telescope Science Institute, which is operated by the Association of Universities for Research in Astronomy, Inc. under NASA contract number NAS5-26555.

${ }^{2}$ Instituto de Física de Cantabria (CSIC-UC), Facultad de Ciencias, Avenida de Los Castros S/N, 39005 Santander, Spain; colina@ifca.unican.es.

${ }^{3}$ Instituto de Astrofísica de Canarias, Vía Láctea S/N, 38200 La Laguna, Tenerife, Spain; sam@11.iac.es.

${ }^{4}$ Consejo Superior de Investigaciones Científicas.

${ }^{5}$ Raytheon Information Technology and Sciences Services, NASA Goddard Space Flight Center, Greenbelt, MD 20771; borne@rings.gsfc.nasa.gov.
}

some ULIRGs is already known to depend on the size of the aperture (Kim, Veilleux, \& Sanders 1998). Two-dimensional optical spectroscopy using fiber-fed spectrographs is an ideal technique to study the complex stellar and ionized gas structure of the nuclear regions of galaxies in general (Arribas \& Mediavilla 2000) and of ULIRGs in particular, since it allows a simultaneous and complete mapping of the stellar populations and the ionized gas (e.g., see Colina, Arribas, \& Borne 1999).

IRAS $12112+0305$ is a low-redshift $(z=0.0723)$ ULIRG $\left(\log L_{\text {IR }}=12.34 L_{\odot}\right)$, classified in the optical as a LINER (Kim et al. 1998) and with no evidence for a hidden broad-line region (Veilleux, Sanders, \& Kim 1999b). Mid-infrared diagnostics classify this galaxy as starburst dominated (Genzel et al. 1998). Near-infrared ground-based images revealed the presence of two nuclei (Carico et al. 1990), while Hubble Space Telescope (HST) optical and near-infrared images show a well-defined compact pointlike nucleus as well as several much fainter condensations distributed in an arclike structure (K. D. Borne, H. Bushouse, L. Colina, \& R. A. Lucas 2000, in preparation; Scoville et al. 2000).

In this Letter, we highlight new results for IRAS $12112+0305$ obtained using integral field optical spectroscopy and HST imaging. Throughout the Letter, a Hubble constant of $70 \mathrm{~km} \mathrm{~s}^{-1} \mathrm{Mpc}^{-1}$ is assumed.

\section{OBSERVATIONS AND DATA REDUCTION}

Integral field spectroscopy of IRAS $12112+0305$ was obtained with the INTEGRAL system (Arribas et al. 1998), a fiber-fed spectrograph mounted at the Naysmith number 1 platform of the $4.2 \mathrm{~m}$ William Herschel Telescope. The bundle of fibers consisted of 219 fibers, each 0 ".9 in diameter and covering a $16 . .5 \times 12$ ".3 field of view. The spectra were taken using a 600 line $\mathrm{mm}^{-1}$ grating, covering the 5000-7900 $\AA$ range, with an effective resolution of $4.8 \AA$. The total integration time was $7500 \mathrm{~s}$, split into five separate integrations of $1500 \mathrm{~s}$ each, with seeing $\approx 1$ ".0.

The reduction has been done following the standard procedures applied to spectra obtained with two-dimensional fiber spectrographs (Arribas et al. 1997 and references therein). The 

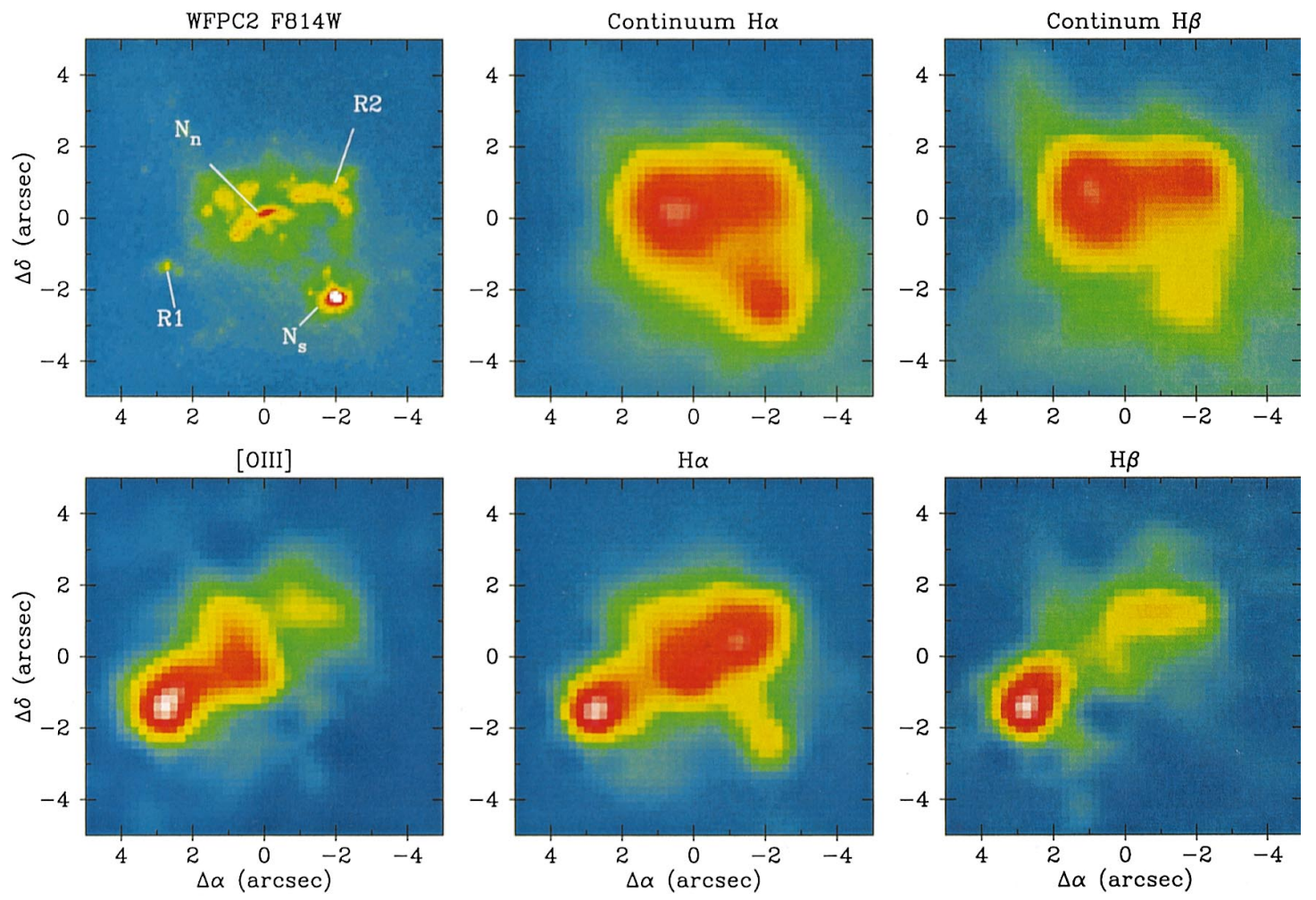

FIG. 1.-INTEGRAL images of the ionized gas and stellar light distribution in the central regions of IRAS $12112+0305$ as traced by different emission lines $(\mathrm{H} \beta,[\mathrm{O} \mathrm{III}] \lambda 5007$, and $\mathrm{H} \alpha)$ and by continuum windows close to $\mathrm{H} \beta$ and $\mathrm{H} \alpha$. The high-resolution $H S T$ I-band image is also shown for comparison. The ionized gas morphology does not follow the stellar light distribution. The southern nucleus $\left(\mathrm{N}_{\text {south }}\right)$, associated with the pointlike $I$-band region seen in the $H S T$ image, appears as a low surface brightness emission peak in $\mathrm{H} \alpha$. The emission line gas is dominated by a region (R1) located $5^{\prime \prime}(\approx 7.5 \mathrm{kpc})$ east-northeast of $\mathrm{N}_{\text {south }}(\mathrm{see}$ text for details).

results are presented in Figure 1 together with the HST I-band image (K. D. Borne, H. Bushouse, L. Colina, \& R. A. Lucas 2000 , in preparation) for comparison.

The derived astrophysical properties for the main continuum and line-emitting regions are presented in Table 1. Columns (2) and (3) give the relative positions. Column (4) presents the internal reddening values derived from the $\mathrm{H} \alpha / \mathrm{H} \beta$ ratio, assuming case B recombination. Column (5) gives the apparent magnitude within an aperture of 0.5 radius using the $H S T I$ band image, and column (6) gives the corresponding absolute magnitude after internal reddening correction assuming $A_{I}=$ $1.494 E(B-V)$. Column (7) shows the observed $\mathrm{H} \alpha$ flux obtained using an aperture of 2 ". 1 diameter, while column (8) gives the reddening-corrected $\mathrm{H} \alpha$ luminosity. The last four columns present the logarithm of the reddening-corrected emission-line ratios and the corresponding activity classification.

\section{RESULTS AND DISCUSSION}

\subsection{Evidence for Decoupled Stellar and Ionized Gas Components}

The stellar main body of IRAS $12112+0305$ is concentrated in three dominant regions, each separated from each other by about $2^{\prime \prime}-3^{\prime \prime}$ (see the $\mathrm{H} \alpha$ and $\mathrm{H} \beta$ continuum images in Fig. 1). Two of these regions (called $\mathrm{N}_{\text {south }}$ and $\mathrm{N}_{\text {north }}$ hereafter; see Table 1 for relative positions) located along a position angle (P.A.) of $45^{\circ}$, were already detected in the near-infrared and associated with the nuclei of two galaxies involved in the final stages of a merger (Carico et al. 1990). The INTEGRAL-generated continuum images extend the wavelength coverage toward the blue, clearly showing the presence of a large differential extinction toward the southern nucleus $\left(\mathrm{N}_{\text {south }}\right)$. The third region

TABLE 1

Properties of the Emission-Line Regions

\begin{tabular}{|c|c|c|c|c|c|c|c|c|c|c|c|}
\hline $\begin{array}{c}\text { Region } \\
\text { (1) }\end{array}$ & $\begin{array}{c}\triangle \alpha^{\mathrm{a}} \\
(2)\end{array}$ & $\begin{array}{l}\triangle \delta^{\mathrm{a}} \\
(3)\end{array}$ & $\begin{array}{c}E(B-V) \\
(4)\end{array}$ & $\begin{array}{l}m_{I}^{\mathrm{b}} \\
(5)\end{array}$ & $\begin{array}{l}M_{I} \\
(6)\end{array}$ & $\begin{array}{c}F_{\text {obs }}(\mathrm{H} \alpha)^{\mathrm{c}} \\
(7)\end{array}$ & $\begin{array}{l}L(\mathrm{H} \alpha)^{\mathrm{d}} \\
\quad(8)\end{array}$ & $\begin{array}{c}{[\mathrm{O} \mathrm{III}] / \mathrm{H} \beta} \\
(9)\end{array}$ & $\begin{array}{c}{\left[\mathrm{O}_{\mathrm{I}}\right] / \mathrm{H} \alpha} \\
\quad(10)\end{array}$ & $\begin{array}{c}{\left[\mathrm{N}_{\mathrm{II}}\right] / \mathrm{H} \alpha} \\
(11)\end{array}$ & $\begin{array}{c}{[\mathrm{S} \text { II }] / \mathrm{H} \alpha} \\
\quad(12)\end{array}$ \\
\hline $\mathrm{N}_{\text {south }} \ldots \ldots$ & -2.0 & -2.2 & 2.62 & 18.61 & -22.8 & 1.75 & 83.9 & 0.25 & $-0.88(\mathrm{w}-\mathrm{L})$ & $-0.15(\mathrm{~L})$ & $-0.50(\mathrm{H} / \mathrm{L})$ \\
\hline $\mathrm{N}_{\text {north }} \ldots \ldots$ & 0.0 & 0.0 & 1.13 & 18.96 & -20.2 & 4.43 & 6.9 & 0.41 & $-0.85(\mathrm{w}-\mathrm{L})$ & $-0.31(\mathrm{H} / \mathrm{L})$ & $-0.31(\mathrm{~L})$ \\
\hline $\mathrm{R} 1 \ldots \ldots$. & 2.7 & -1.5 & 0.31 & 20.35 & -17.7 & 3.67 & 0.9 & 0.25 & $-1.35(\mathrm{H})$ & $-0.66(\mathrm{H})$ & $-0.63(\mathrm{H})$ \\
\hline $\mathrm{R} 2 \ldots \ldots \ldots$ & -1.5 & 0.6 & 1.11 & 19.16 & -20.0 & 4.40 & 6.5 & -0.02 & $-1.05(\mathrm{w}-\mathrm{L})$ & $-0.41(\mathrm{H})$ & $-0.34(\mathrm{~L})$ \\
\hline
\end{tabular}

Note.-L: LINER; H: H II region; w-L: weak [O I] LINER with $I([\mathrm{O}$ I] $]) /\left((\mathrm{H} \alpha)<\frac{1}{6}\right.$; H/L: boundary of H II and LINER.

${ }^{a}$ Relative positions in arcseconds as measured from the WFPC2 I-band image (see Fig. 1).

${ }^{\mathrm{b}}$ Uncertainties of $\pm 0.05 \mathrm{mag}$.

${ }^{c}$ Flux in units of $10^{-15} \mathrm{ergs} \mathrm{s}^{-1} \mathrm{~cm}^{-2} \AA^{-1}$. Uncertainties of $10 \%-15 \%$.

${ }^{\mathrm{d}}$ Reddening-corrected luminosity in units of $10^{41} \mathrm{ergs} \mathrm{s}^{-1}$. 
(called R2 hereafter) is located $3^{\prime \prime}$ north of $\mathrm{N}_{\text {south }}$ at P.A. = $20^{\circ}$.

The HST I-band image (Fig. 1) shows $\mathrm{N}_{\text {south }}$ as a high surface brightness compact region that coincides with the $K$-band pointlike nucleus (Scoville et al. 2000). Regions $\mathrm{N}_{\text {north }}$ and $\mathrm{R} 2$ are made of several fainter condensations distributed along an arclike structure of about 5".4 (i.e., $8 \mathrm{kpc}$ ) extent and located $3^{\prime \prime}-5^{\prime \prime}$ (i.e., $4.5-7.5 \mathrm{kpc}$ ) north-northeast of $\mathrm{N}_{\text {south }}$. The brightest of these condensations, located $3^{\prime \prime}(\approx 4.5 \mathrm{kpc})$ northeast from $\mathrm{N}_{\text {south }}$, is most likely associated with the northern nucleus detected in the INTEGRAL-generated continuum images $\left(\mathrm{N}_{\text {north }}\right)$ and in the near-infrared (Carico et al. 1990; Scoville et al. 2000).

Although the overall structure of the ionized gas resembles that of the stellar light distribution, the dominant line-emitting regions do not coincide with the nuclei identified above but, on the contrary, are decoupled from them (see [O III] and $\mathrm{H} \alpha$ maps in Fig. 1). The brightest line-emitting region (called R1 hereafter) is located $5^{\prime \prime}(\approx 7.5 \mathrm{kpc})$ east of $\mathrm{N}_{\text {south }}$ along P.A. = $80^{\circ}$. This region is associated with a faint $I$-band continuum source $\left(m_{I}=20.4\right)$ outside the main body of the galaxy (see HST I-band image in Fig. 1). The second brightest $\mathrm{H} \alpha$ lineemitting region is associated with region $\mathrm{R} 2$, and it is therefore composed of a filament of faint continuum condensations that are also detected in the $\mathrm{H} \beta$ and [O III] maps (Fig. 1). The optically dominant nucleus of the galaxy $\left(\mathrm{N}_{\text {south }}\right)$ is marginally detected in [O $\mathrm{III}]$ and appears as the faintest $\mathrm{H} \alpha$ emission source. The arclike structure seen in the HST I-band image corresponds to three well-resolved $\mathrm{H} \alpha$ line-emitting peaks, the faintest of which is associated with the northern nucleus $\left(\mathrm{N}_{\text {north }}\right)$ detected in the optical and near-infrared (see discussion above).

\subsection{Massive Dust-enshrouded Starbursts as Nuclear Ionizing Sources}

The positions of the two apparently faint ionizing nuclei $\mathrm{N}_{\text {north }}$ and $\mathrm{N}_{\text {south }}$ coincide (within the HST absolute astrometry uncertainties of $\left.\pm 0.7-1^{\prime \prime}\right)$ with the two bright compact starburst nuclei detected at radio frequencies (Condon et al. 1991), thus favoring dust-enshrouded nuclear starbursts as the energy sources in these nuclei.

The ionization sources in $\mathrm{N}_{\text {south }}$ and $\mathrm{N}_{\text {north }}$ are highly obscured, with visual extinctions $\left(A_{V}\right)$ of 8 and $3.5 \mathrm{mag}$, respectively (see spectra in Fig. 2). The two nuclei appear almost equally bright with $I$-band absolute magnitudes of $-18.9\left(\mathrm{~N}_{\text {south }}\right)$ and -18.5 $\left(\mathrm{N}_{\text {north }}\right)$. However, when reddening is taken into account, $\mathrm{N}_{\text {south }}$ is found to be 10 times more luminous than $\mathrm{N}_{\text {north }}$ (i.e., $M_{I}=$ -22.8). These magnitudes are within the range of those measured for the nuclei of other luminous and ultraluminous infrared galaxies (Surace et al. 1998).

The line ratios of the ionization sources associated with the nuclei $\mathrm{N}_{\text {south }}$ and $\mathrm{N}_{\text {north }}$ correspond to a mixture of weak [O I] LINER and $\mathrm{H}$ II region spectra (see Table 1). The presence of weak [O I] LINERs in the nucleus of galaxies has been taken as evidence for ionization by hot stars in a high-metallicity environment (Filippenko \& Terlevich 1992; Shields 1992) or for ionization by a mixture of a low-luminosity active galactic nucleus (AGN) and hot stars (Ho, Filippenko, \& Sargent 1993). The integrated mid-infrared spectrum of IRAS $12112+0305$ does not show any evidence for an AGN (Genzel et al. 1998), further supporting the idea that the ionizing sources in the nuclei $\mathrm{N}_{\text {south }}$ and $\mathrm{N}_{\text {north }}$ are associated with dust-enshrouded starbursts. Although $\mathrm{N}_{\text {south }}$ and $\mathrm{N}_{\text {north }}$ are minor contributors to the observed $\mathrm{H} \alpha$ emission, the reddening-corrected flux emanating from these nuclei dominates the overall $\mathrm{H} \alpha$ luminosity with a

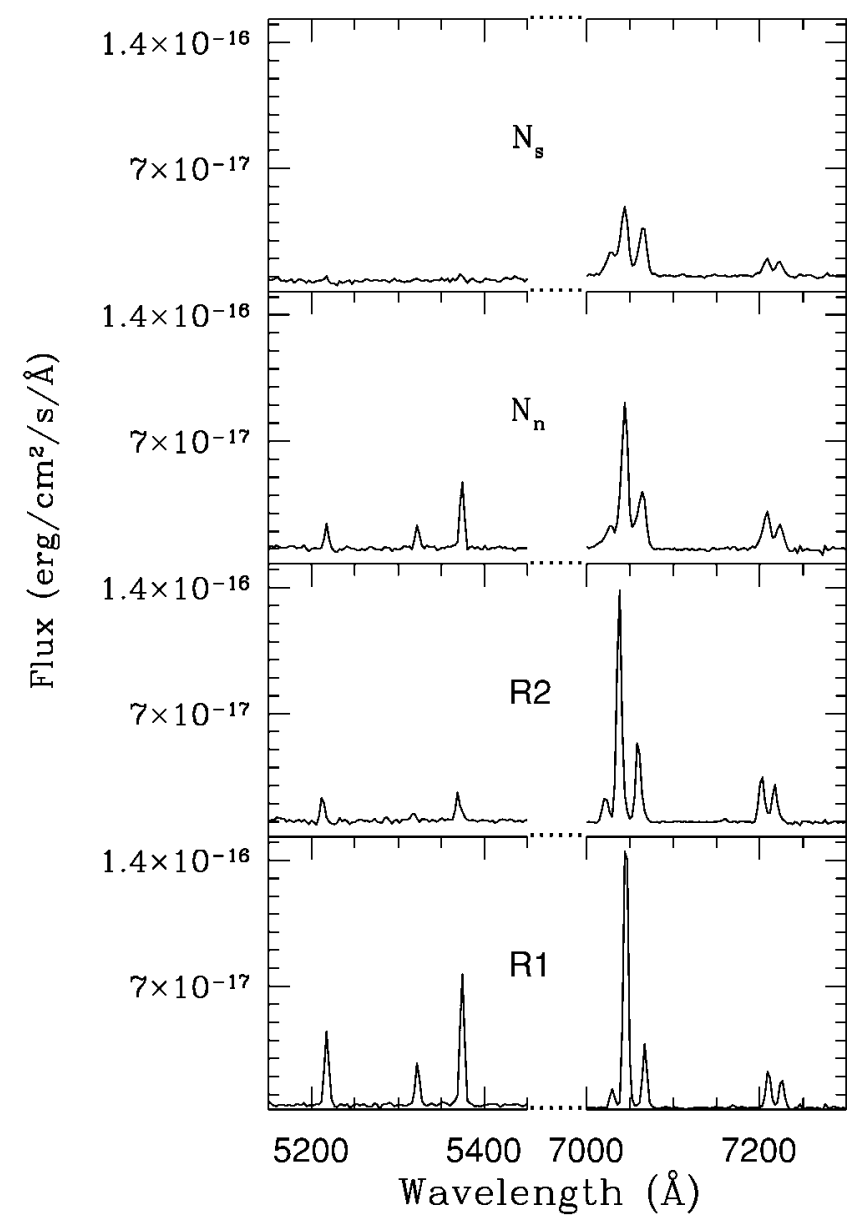

FIG. 2.-Plots showing spectra of the main line-emitting regions (R1 and R2) and of the northern and southern nuclei $\left(\mathrm{N}_{\text {north }}\right.$ and $\mathrm{N}_{\text {south }}$, respectively). Note that the horizontal axis does not represent a continuous range in wavelength, but the plots present two subsets of the full INTEGRAL spectrum corresponding to the $\mathrm{H} \beta-[\mathrm{O} \mathrm{III}]$ and $\mathrm{H} \alpha-[\mathrm{S}$ II $]$ spectral regions, respectively.

value of $9.1 \times 10^{42} \mathrm{ergs} \mathrm{s}^{-1}$ (Table 1). If the $\mathrm{H} \alpha$ flux emitted by the two nuclei were entirely due to stars, then the corresponding star formation rate would amount to about $80 M_{\odot}$ $\mathrm{yr}^{-1}$ for a Salpeter initial mass function (IMF) with mass limits of 0.1 and $100 M_{\odot}$ (Leitherer et al. 1999).

\subsection{Brightest Line-emitting Region: A Tidally Induced Giant H II Region?}

The HST image (Fig. 1) shows that the apparently more luminous line-emitting peak (R1) is associated with a faint $\left(m_{I}=20.35\right)$ region characterized by a physical size of about 600 pc $(\approx 0.4)$, an $I$-band absolute magnitude of -17.7 , an internal optical extinction of about $1 \mathrm{mag}$, and an $\mathrm{H} \alpha$ luminosity of $8.7 \times 10^{40} \mathrm{ergs} \mathrm{s}^{-1}$ (see Table 1 ).

The size, $\mathrm{H} \alpha$ luminosity, and emission-line ratios are typical of circumnuclear star-forming regions in nearby spiral galaxies (Gonzalez-Delgado \& Pérez 1997; Planesas, Colina, \& PérezOlea 1997), and of giant extragalactic $H$ II regions like NGC 5471 in M101 (Shields 1990). The H $\alpha$ luminosity and the equivalent widths of the $\mathrm{H} \beta(74 \pm 3 \AA)$ and $\mathrm{H} \alpha(450 \pm$ $10 \AA$ ) emission lines correspond to that of a 5 Myr old ionizing cluster of $2 \times 10^{7} M_{\odot}$, assuming a Salpeter IMF with mass limits of 0.1 and $100 M_{\odot}$ (Leitherer et al. 1999). The mass of the ionizing cluster represents only a small fraction $(\sim 3 \%)$ of 
the dynamical mass of this region (upper limit of $7.5 \times 10^{8}$ $M_{\odot}$ ), calculated assuming virialization and an instrumentcorrected emission line width equivalent to a velocity dispersion of $60 \mathrm{~km} \mathrm{~s}^{-1}$ (derived from the line-width measurements of the $\mathrm{H} \beta$ and $[\mathrm{O} \mathrm{III}] \lambda 5007$ lines).

In summary, the derived properties of this region are characteristic of young massive $\mathrm{H}$ II regions and could represent a case for a tidally induced giant extranuclear self-gravitating star-forming region, or even a dwarf galaxy decoupled from the much older stellar body of the parent galaxies (Duc \& Mirabel 1994, 1998).

\section{SUMMARY}

Integral field optical spectroscopy and HST imaging have revealed the complex stellar and ionized gas structures in the ultraluminous infrared galaxy IRAS $12112+0305$. The two nuclei detected in the optical coincide with previously known radio and near-infrared nuclei, but appear as the apparently two faintest line-emitting regions and are consistent with dustenshrouded ( $A_{V}=3.5$ and 8 mag) weak [O I] LINERs. However, their reddening-corrected $\mathrm{H} \alpha$ emission dominates the overall $\mathrm{H} \alpha$ luminosity of the system and, if associated with nuclear starbursts, would correspond to a star formation of 80 $M_{\odot} \mathrm{yr}^{-1}$.
The observed structure of the ionized gas is decoupled from, and hence does not trace, the stellar light distribution. The brightest line-emitting peak is associated with a faint $\left(m_{I}=\right.$ 20.4 ) region located $7.5 \mathrm{kpc}$ from the dominant optical nucleus $\left(\mathrm{N}_{\text {south }}\right)$, well outside the main stellar body of the system. This region appears to be a recent (tidally induced?) star-forming region containing a young $(5 \mathrm{Myr})$, massive $\left(2 \times 10^{7} M_{\odot}\right)$ cluster of stars.

The results presented here for IRAS $12112+0305$ stress the need for integral-field spectrographs in the study of the complex ionization and stellar light structure of ULIRGs. Similar studies for high-redshift analogs of ULIRGs (i.e., the SCUBA sources), and for other morphologically complex high-redshift galaxies, will become feasible when integral-field spectrographs on giant telescopes become operational.

L. C. thanks the Instituto de Astrofísica de Canarias for its hospitality and financial support. K. D. B. thanks Raytheon for providing financial support during his sabbatical leave. Support for this work was provided by CICYT (Comisión Interministerial de Ciencia y Tecnología) through grants PB98-0340-C0201 and PB98-0340-C02-02 and by NASA through grant GO06346.01-95A from the Space Telescope Science Institute, which is operated by AURA, Inc., under NASA contract NAS526555 .

\section{REFERENCES}

Arribas, S., et al. 1998, Proc. SPIE, 3355, 821

Arribas, S., \& Mediavilla, E. 2000, in Imaging the Universe in Three Dimensions: Astrophysics with Advanced Multiwavelength Imaging Devices (San Francisco: ASP), in press

Arribas, S., Mediavilla, E., García-Lorenzo, B., \& del Burgo, C. 1997, ApJ, 490, 227

Borne, K. D., Bushouse, H., Lucas, R. A., \& Colina, L. 2000, ApJ, 529, L77

Carico, D. P., Graham, J. R., Matthews, K., Wilson, T. D., Soifer, B. T., Neugebauer, G., \& Sanders, D. B. 1990, ApJ, 349, L39

Clements, D. L., Sutherland, W. J., McMahon, R. G., \& Saunders, W. 1996, MNRAS, 279, 477

Colina, L., Arribas, S., \& Borne, K. D. 1999, ApJ, 527, L13

Condon, J. J., Huang, Z. P., Yin, Q. F., \& Thuan, T. X. 1991, ApJ, 378, 65

Duc, P. A., \& Mirabel, I. F. 1994, A\&A, 289, 83 1998, A\&A, 333, 813

Filippenko, A., \& Terlevich, R. 1992, ApJ, 397, L79

Genzel, R., et al. 1998, ApJ, 498, 579

Gonzalez-Delgado, R., \& Pérez, E. 1997, ApJS, 108, 199

Ho, L., Filippenko, A., \& Sargent, W. 1993, ApJ, 417, 63
Kim, D. C., Veilleux, S., \& Sanders, D. B. 1998, ApJ, 508, 627

Leitherer, C., et al. 1999, ApJS, 123, 3

Lutz, D., Spoon, H. W. W., Rigopoulou, D., Moorwood, A. F. M., \& Genzel, R. 1998, ApJ, 505, L103

Lutz, D., Veilleux, S., \& Genzel, R. 1999, ApJ, 517, L13

Melnick, J., \& Mirabel, I. F. 1990, A\&A, 231, L19

Planesas, P., Colina, L., \& Pérez-Olea, D. 1997, A\&A, 325, 81

Sanders, D. B. 2000, in Proc. Conf. Space Infrared Telescopes and Related Science, 32nd COSPAR Meeting, ed. T. Matsumoto \& T. de Graauw, in press (astro-ph/9904292)

Sanders, D., \& Mirabel, I. F. 1996, ARA\&A, 34, 749

Scoville, N. Z., et al. 2000, AJ, in press (astro-ph/9912246)

Shields, G. 1990, ARA\&A, 28, 525

Shields, J. C. 1992, ApJ, 399, L27

Surace, J. A., Sanders, D. B., Vacca, W. D., Veilleux, S., \& Mazzarella, J. M. 1998, ApJ, 492, 116

Veilleux, S., Kim, D. C., \& Sanders, D. B. 1999a, ApJ, 522, 113

Veilleux, S., Sanders, D. B., \& Kim, D. C. 1999b, ApJ, 522, 139 